https://doi.org/10.15407/ujpe63.11.979

O. NOVAK, M. DIACHENKO, E. PADUSENKO, R. KHOLODOV

Institute of Applied Physics, Nat. Acad. of Sci. of Ukraine

(58, Petropavlivs'ka Str., Sumy 40000,Ukraine; e-mail: novak-o-p@ukr.net)

\title{
VACUUM BIREFRINGENCE IN THE FIELDS OF A CURRENT COIL AND A GUIDED ELECTROMAGNETIC WAVE
}

\begin{abstract}
The vacuum birefringence effect in magnetic fields generated by either a laser-driven capacitorcoil generator or an electromagnetic wave in a radio frequency guide has been theoretically studied. The ellipticity acquired by a linearly polarized laser beam propagating in those fields is calculated. The obtained results are compared with the parameters of the PVLAS experiment aimed at the experimental observation of the vacuum birefringence effect in a magnetic field.

Keywords: quantum electrodynamics, strong fields, birefringence, vacuum polarization, magnetic field, laser radiation.
\end{abstract}

\section{Introduction}

The equations of classical electrodynamics are linear, so that there is no interaction of photons with other photons and external fields in the classical theory. The uncertainty ratio, however, allows photons to form virtual electron-positron pairs. Those pairs can interact with fields and other photons. Thus, the quantum theory predicts a number of nonlinear vacuum effects, among which there is a change of the polarization for photons propagating in an external magnetic field. This effect is known as the magnetic vacuum birefringence. It was proposed for the first time by Heisenberg and Euler in work [1]. In the framework of scattering theory, this process is described by the Feynman diagram depicted in Fig. 1. The study of this process in the general case requires the calculation of a polarization operator [2-5]. In the low-energy approximation, $\hbar \omega \ll m c^{2}$, the effective Lagrangian obtained in work [1] is widely used.

A high polarization degree of the optical radiation from the isolated neutron star RX J1856.5-3754 [6] is considered to be the first direct evidence of the vacuum birefringence effect. According to the modern models of pulsar atmosphere, a high polarization of the radiation emitted by it can be explained, only if vacuum effects in superstrong fields of neutron stars are made allowance for [7-12].

\section{(C) O. NOVAK, M. DIACHENKO, E. PADUSENKO, R. KHOLODOV, 2018}

ISSN 2071-0194. Ukr. J. Phys. 2018. Vol. 63, No. 11
Under laboratory conditions, the effect has not been observed yet, because it is too weak for the experimentally attainable fields. The direct observation of a beam polarization change in the magnetic field is the purpose of the PVLAS experiment (Polarizzazione del Vuoto con LASer), which is carried out on the basis of the National Institute of $\mathrm{Nu}$ clear Physics in Ferrara, Italy [13-15]. The method is based on the measurement of the ellipticity acquired by a linearly polarized laser beam with the wavelength $\lambda=1064 \mathrm{~nm}$, when it passes through the distance $L=1.64 \mathrm{~m}$ in vacuum in a magnetic field with the induction $B \approx 2.5 \mathrm{~T}$. A Fabry-Perot resonator is used to enhance the effect: it enlarges the beam path length in the field by a factor of about $10^{5}$. For the indicated parameters, the theoretical value of the induced ellipticity equals $\psi \approx 1.6 \times 10^{-16}$ per passage. Despite a significant progress in the experimental technology, the current accuracy of measurements of the ellipticity is still an order of magnitude lower than that required to register the effect [15].

In this work, the vacuum birefringence effect in electromagnetic fields with various configurations is studied theoretically. Section 2 contains a brief overview of the theory describing a change of the beam polarization in an external field. The ellipticity acquired by a linearly polarized beam in the field of a laser-driven coil generator is calculated in Section 3. In Section 4, a possibility to observe the effect, by using radio frequency waveguides, is discussed. 


\section{Theory}

While studying the evolution of the beam polarization in an external field, it is convenient to apply a method developed in work [16]. In this section, the relativistic unit system is used: $\hbar=c=1$.

From the practical viewpoint, the polarization of photons is described by the Stokes parameters $Q, U$, and $V$. These parameters are closely related to the probability density matrix of photons. The latter has the following form in the basis of linear polarizations:

$\rho=\frac{1}{2}\left(\begin{array}{cc}I+Q & U-i V \\ U+i V & I-Q\end{array}\right)$.

Hence, the evolution of Stokes parameters can be determined, if we know the equation for $\rho$. Note that the matrix $\rho$ can be expressed in terms of the mean value of the photon number operator $\hat{D}_{i j}=\hat{a}_{i}^{+} \hat{a}_{j}$,

$\left\langle D_{i j}(\mathbf{k})\right\rangle=(2 \pi)^{3} 2 \omega \delta^{3}(0) \rho_{i j}(\mathbf{k})$.

where $\omega$ and $\mathbf{k}$ are the frequency and the wave vector, respectively, of a photon. In turn, the evolution of the photon number operator is determined by the interaction Hamiltonian [17],

$\left\langle\frac{d \hat{D}_{i j}}{d t}\right\rangle \approx i\left\langle\left[\hat{\mathcal{H}}_{\mathrm{int}}, \hat{D}_{i j}\right]\right\rangle$.

This expression was obtained in the lowest approximation order in the interaction of photons with the field $\hat{\mathcal{H}}_{\text {int }}$. The right-hand side of Eq. (3) describes only a change of the photon polarization, whereas the terms corresponding to scattering processes were neglected.

In the low-energy approximation, it is expedient to use the effective Euler-Heisenberg Lagrangian [1]

$$
\begin{aligned}
& \mathcal{L}_{\text {int }}=-\frac{\alpha^{2} /(4 \pi)^{2}}{180 m^{4}}\left[5\left(\mathcal{F}_{\mu \nu} \mathcal{F}^{\mu \nu}\right)^{2}-\right. \\
& \left.-14 \mathcal{F}_{\mu \nu} \mathcal{F}^{\nu \lambda} \mathcal{F}_{\lambda \sigma} \mathcal{F}^{\sigma \mu}\right],
\end{aligned}
$$

where $\alpha$ is the fine structure constant, and $m$ the electron mass.

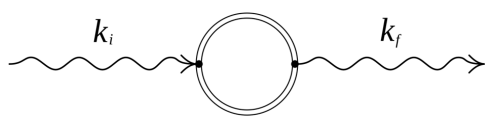

Fig. 1. Feynman diagram for the process of photon polarization change in a magnetic field. Double lines denote an electron propagator in the external field
In order to consider the interaction of photons with an external field, let us represent the electromagnetic field tensor in Eq. (4) in the form $\mathcal{F}^{\mu \nu}=F^{\mu \nu}+f^{\mu \nu}$, where $F^{\mu \nu}$ is a slowly changing external field, and the quantum field $f^{\mu \nu}$ plays the role of a small perturbation in the Hamiltonian. In order to use Eq. (3), the tensor $f^{\mu \nu}$ has to be expressed in terms of the photon creation and annihilation operators $\hat{a}$ and $\hat{a}^{+}$, which enter the 4-potential $A_{\nu}$ :

$f^{\mu \nu}=\partial_{\mu} \hat{A}_{\nu}-\partial_{\nu} \hat{A}_{\mu}$

$\hat{A}^{\nu}=\sqrt{4 \pi} \int \frac{d^{3} k /(2 \pi)^{3}}{\sqrt{2 \omega}} \times$

$\times \sum_{s=1,2}\left[\hat{a}_{\mathbf{k} s} e_{s}^{\nu} \mathrm{e}^{-i k r}+\hat{a}_{\mathbf{k} s}^{+} e_{s}^{\nu *} \mathrm{e}^{i k r}\right]$

where $e_{1,2}=\left(0, \mathbf{e}_{1,2}\right)$ is the photon polarization 4 vector.

Using expressions (1)-(6) and making simple transformations, we obtain a system of differential equations for the Stokes parameters. It is convenient to rewrite this system in the vector form by introducing the Stokes vector $\mathbf{S}=(Q, U, V)$ :

$\dot{\mathbf{S}}=[\mathbf{\Omega} \times \mathbf{S}]$,

$\Omega_{x}=-G\left(\varepsilon_{2}^{2}-\varepsilon_{1}^{2}\right)$,

$\Omega_{y}=2 G \varepsilon_{1} \varepsilon_{2}$,

$\Omega_{z}=0$.

Here, the notations

$G=\frac{\alpha^{2}}{4 \pi} \frac{2 \omega}{15 m^{4}}$

$\varepsilon_{i}=\mathbf{E e}_{i}-\mathbf{B}\left[\mathbf{n} \times \mathbf{e}_{i}\right], \quad i=x, y$,

are introduced; $\mathbf{E}$ and $\mathbf{B}$ are the vectors of electric strength and magnetic induction, respectively, of the external field; and $\mathbf{n}=\mathbf{k} / k$ is a unit vector along the wave propagation direction. Equation (7) was also obtained in work [18] and analyzed in detail for a constant uniform external field [19, 20]. The numerical solution of Eq. (7) makes it possible to determine the polarization of photons in a slowly varying electromagnetic field with an arbitrary configuration.

\section{Laser-Driven Coil Generator}

As follows from Eqs. (7)-(10), the magnitude of the vacuum birefringence effect is proportional to

ISSN 2071-0194. Ukr. J. Phys. 2018. Vol. 63, No. 11 
the squared induction of an external magnetic field. Hence, a natural way to experimentally detect this effect consists in increasing the field strength. Under laboratory conditions, the strongest stationary field with an induction of about $45 \mathrm{~T}$ was obtained with the help of hybrid superconducting magnets [21]. The non-destructive methods of generating pulsed fields enable fields of about $100 \mathrm{~T}$ within a few microseconds to be obtained. Recently, laserdriven coil generators of magnetic field attract a considerable interest. They make it possible to generate field pulses with an induction of several hundred teslas and a duration of a few nanoseconds [22-27].

The generator is a single conducting coil connected to a capacitor-target (Fig. 2). One of the target plate is irradiated with a powerful laser pulse. The resonant absorption of radiation leads to a strong heating of electrons, which are emitted from one capacitor plate onto another and create a significant voltage drop between the plates. The electric current runs along the coil and creates a strong magnetic field. The authors of work [27] reported on the observation of a peak magnetic field with an induction of $610 \pm 30 \mathrm{~T}$ in experiments using a generator with a coil $0.5 \mathrm{~mm}$ in diameter.

Let us estimate the ellipticity induced by the vacuum birefringence effect in a possible experiment with a similar field generator. For simplicity, we assume the field to be quasistationary. This assumption is based on the fact that the time interval required for the beam to cross characteristic field dimensions amounts to picoseconds, whereas the field lifetime is three orders of magnitude longer. Let us assume that the generator is a coil with current and use a cylindrical coordinate frame $(\rho, \phi, z)$ with the origin at the coil center and the axis $O z$ directed perpendicularly to the coil plane. The coil radius is $R=0.5 \mathrm{~mm}$, and the induction at its center $B_{0}=500 \mathrm{~T}$. The field created by the coil with the current at an arbitrary spatial point $(\rho, \phi, z)$ is determined in terms of the coil radius and the induction at the coil center as follows:

$B_{\rho}=\frac{B_{0} R}{\pi} \frac{z}{u^{2} w \rho}\left[\left(z^{2}+\rho^{2}+R^{2}\right) E\left(q^{2}\right)-u^{2} K\left(q^{2}\right)\right]$,

$B_{\varphi}=0$,

$B_{z}=\frac{B_{0} R}{\pi} \frac{1}{u^{2} w}\left[\left(R^{2}-z^{2}-\rho^{2}\right) E\left(q^{2}\right)+u^{2} K\left(q^{2}\right)\right]$, where the notations

$q^{2}=1-u^{2} / w^{2}$

$u=z^{2}+(R-\rho)^{2}$,

$w=z^{2}+(R+\rho)^{2}$

were introduced.

Equations (7) were numerically integrated for the external field (11). The initial laser beam polarization was chosen linear with the Stokes parameters

$\mathbf{S}_{0}=(0,1,0)$

i.e. the beam polarization plane formed an angle of $45^{\circ}$ with the axes of the coordinate system, in which the polarization is determined.

Having passed through the external field, the linearly polarized beam acquires a non-zero circular polarization $V \neq 0$. In practice, it is convenient to characterize the polarization by the ellipticity parameter $K$. In the case $U=1$ and $V \ll 1$, it can be determined as

$K \approx 2 V$.

Figure 3 illustrates the resulting ellipticity of beams propagating perpendicularly to the coil plane. The picture is symmetric with respect to its rotation by $180^{\circ}$. Really, Eqs. (7)-(10) are quadratic in the field, so they do not change at the substitution $\mathbf{B} \rightarrow-\mathbf{B}$. From Eqs. (10), it also follows that the contribution is made only by the field components that are perpendicular to the beam wave vector. Figure 3 confirms that the effect is absent for a beam propagating through the coil center. The most pronounced effect is observed near the coil wire, but this situation is unfavorable for the experimental effect observation, because the coil is being destroyed in the course of the experiment, and the region near it becomes filled

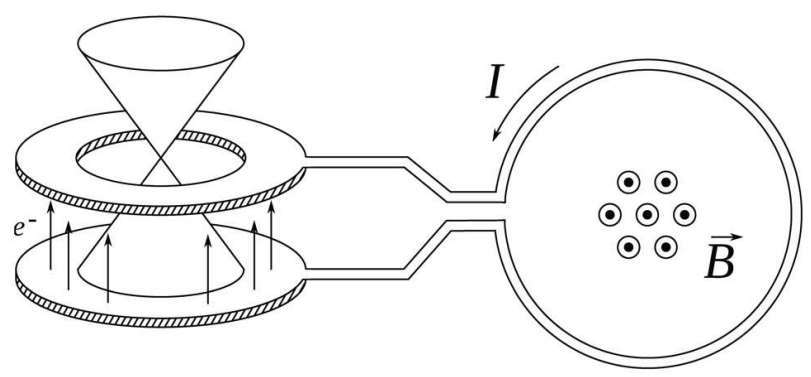

Fig. 2. Schematic diagram of a laser-driven coil generator 


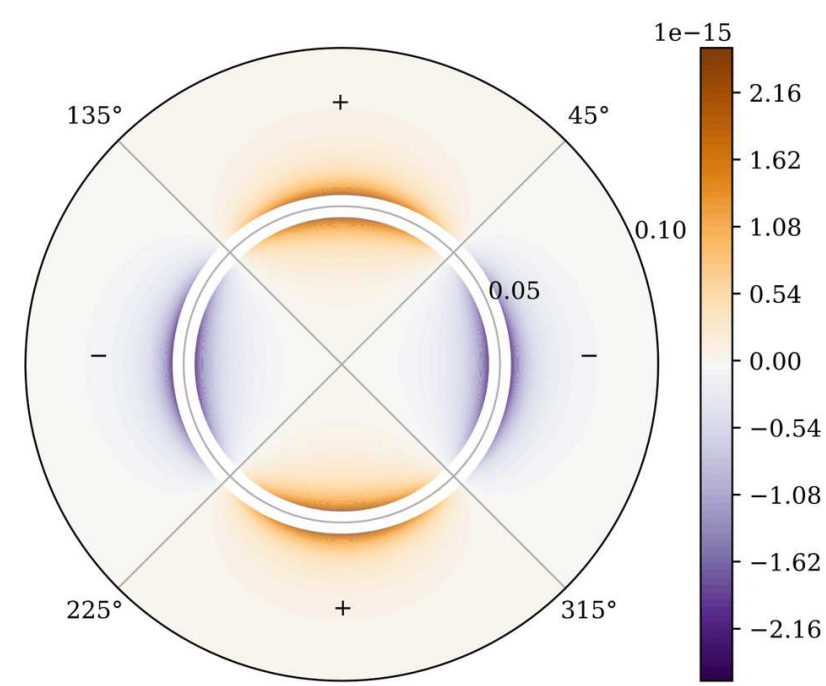

Fig. 3. Ellipticity of beams propagating perpendicularly to the coil plane

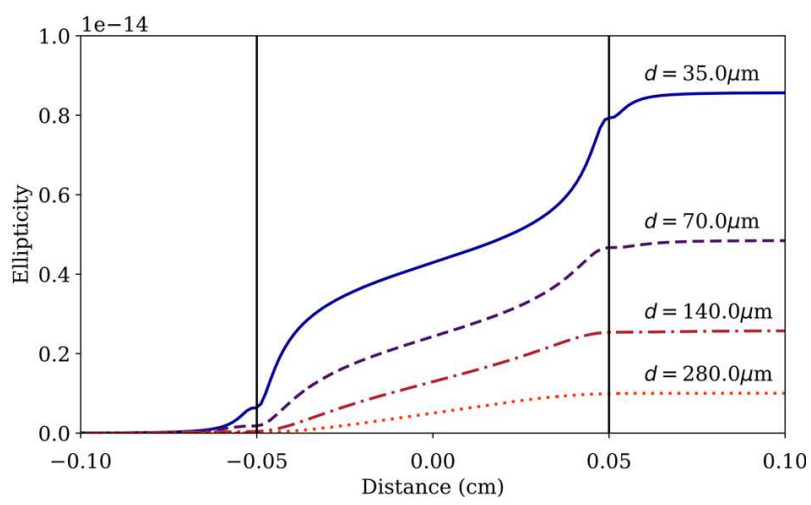

Fig. 4. Dependence of the beam ellipticity on the distance passed in the coil field. The beam propagates in parallel to the coil at the distance $d$ from its plane and across the coil axis. Vertical lines mark the coil position

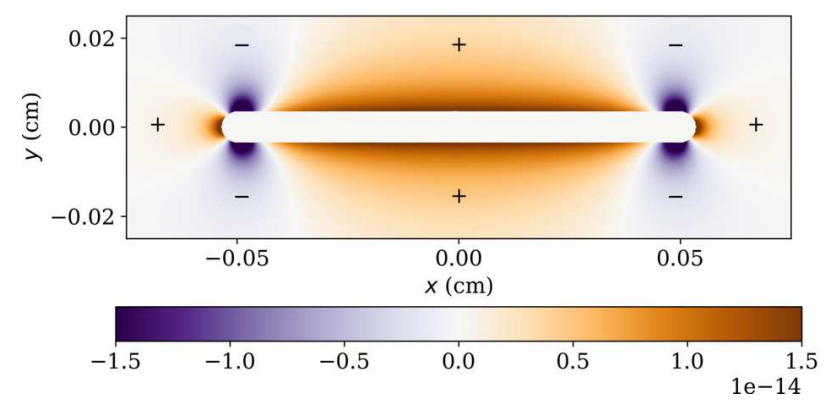

Fig. 5. Ellipticity of beams propagating in parallel to the coil plane

982 with a plasma, which inevitably gives rise to a result uncertainty.

More expedient is a configuration, in which the beam propagates in parallel to the coil plane at the distance $d$ from it. Figure 4 exhibits the final ellipticity of the beams in this case. Note that the effect can be enhanced by applying two generators in the Helmholtz configuration, as was done in work [27]. Figure 5 depicts the corresponding ellipticity evolution of the beam with the distance passed by the beam in the coil field for various values of the parameter $d$. As one can see, the magnitude of the effect can reach a value of $10^{-15}$, which is an order of magnitude larger than in the PVLAS experiment $[14,15]$.

Note that, in the PVLAS experiment [14, 15], a Fabry-Perot resonator with the gain factor $N \sim 10^{5}$ is used in order to increase the beam path length in the field. The pulsed character of a laser-driven generator field can restrict possible values of the gain factor. Really, let us suppose that the interferometer size is compared with the coil size by the order of magnitude. Then the time of the passage of a beam through a resonator is about $10^{-12} \mathrm{~s}$. Taking into account that the field lifetime amounts to a few nanoseconds, we obtain that the maximum gain factor also reaches $10^{5}$.

Thus, a conclusion can be drawn that the application of laser-driven coil generator in experiments aimed at the observation of the vacuum birefringence can increase the effect by an order of magnitude. On the other hand, the destructive behavior of a generator may complicate such experiments and reduce their accuracy.

Let us evaluate the residual atmosphere pressure $p$, at which the vacuum birefringence effect has the same magnitude as the similar Cotton-Mouton effect in gases. The ellipticity associated with the latter can be found from the expression $[15,28]$

$K=\frac{\pi L}{\lambda}\left(\frac{B}{1 \mathrm{~T}}\right)^{2}\left(\frac{p}{1 \mathrm{~atm}}\right) \Delta n_{u}$

where $\Delta n_{u}$ is the effect magnitude at a pressure of $1 \mathrm{~atm}$ and a field induction of $1 \mathrm{~T}$. The $\Delta n_{u}$-values were measured experimentally [28, 29]. For example, $\Delta n_{u} \approx 4.3 \times 10^{-15}$ for argon. Then, for typical problem parameters, we have $p \sim 10^{-9}$ atm. Note that a similar evaluation for the PVLAS experimental conditions gives the same value. 


\section{Radio Frequency Waveguide}

Recently, a possibility to observe the vacuum birefringence effect in a laser wave field has become of considerable interest. Modern laser installations can produce magnetic fields, whose strengths far exceed the values reachable under laboratory conditions. In the framework of the Extreme Light Infrastructure (ELI) project, the creation of even more powerful lasers is planned, whose fields should reach a critical quantum-electrodynamic value of about $10^{16} \mathrm{~V} / \mathrm{cm}$.

In this paper, we will consider an alternative experimental setup, in which the role of an external field is played by an electromagnetic wave propagating in a rectangular hollow waveguide (Fig. 6). Currently, the most powerful sources of microwave radiation are klystrons. In particular, in the SLAC National Accelerator Laboratory (USA), klystrons with an operating frequency of $11.424 \mathrm{GHz}$ and a peak power of 75 MW have been built [30].

It is known [31] that the field $\mathcal{F}$ in a waveguide exists in the form of standing waves in transverse directions and a running wave in the longitudinal direction:

$\mathcal{F}=\operatorname{Re} \mathbf{F}(x, y) \mathrm{e}^{i(\omega t-\Gamma z)}$.

The transverse wave numbers are determined by the waveguide geometry,

$$
\begin{aligned}
& \chi^{2}=\chi_{x}^{2}+\chi_{y}^{2}, \\
& \chi_{x}=\frac{m \pi}{a}, \quad \chi_{y}=\frac{n \pi}{b},
\end{aligned}
$$

where $a$ and $b$ are the lengths of waveguide sides, and $m$ and $n$ are integer numbers. The longitudinal wave number $\Gamma$ is determined as

$\Gamma^{2}=\left(\frac{\omega}{c}\right)^{2}-\chi^{2}$

The frequency $\omega_{c}$, at which $\Gamma=0$, is called critical. For frequencies lower $\omega_{c}$, the longitudinal wave number becomes imaginary, and the propagation of the wave is impossible (the cutoff phenomenon).

A specific feature of the waves propagating in hollow waveguides is the presence of the longitudinal component of their electric or magnetic field. Accordingly, the corresponding waves are classed to the $H$ or $E$-type. The numbers $m$ and $n$, which determine the transverse wave number, are usually indicated as subscripts near the mode type. The lowest frequencies

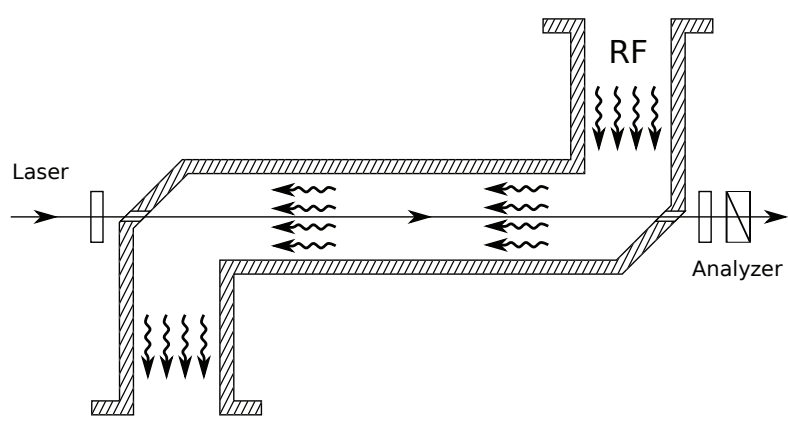

Fig. 6. Application of a radio frequency waveguide as the source of an external field

are characteristic of the $\mathrm{H}_{01}$ and $\mathrm{H}_{10}$ waves, which are called the main ones.

The transverse field components of the $\mathrm{H}$-wave are determined by the following formulas:

$\left\{\begin{array}{l}E_{x}=-F_{0} \frac{\chi_{y}}{\chi} \cos \chi_{x} x \sin \chi_{y} y \\ E_{y}=-F_{0} \frac{\chi_{x}}{\chi} \sin \chi_{x} x \cos \chi_{y} y \\ B_{x}=-F_{0} \frac{\chi_{x}}{\chi} W_{E} \sin \chi_{x} x \cos \chi_{y} y \\ B_{y}=-F_{0} \frac{\chi_{y}}{\chi} W_{E} \cos \chi_{x} x \sin \chi_{y} y\end{array}\right.$

where $W_{E}$ is the wave impedance.

The field amplitude $F_{0}$ in the presented equations can be determined from the energy flux $\bar{P}$ through the waveguide cross-section,

$F_{0}^{2}=\bar{P} \frac{4 \pi}{c} \frac{W_{H}}{a b} \delta_{0}$,

where $\delta_{0}=4$ for the main wave, and $\delta_{0}=8$ for all others. In the case of a waveguide not filled with an insulator, the following relations are valid for the wave impedances $W_{H}$ and $W_{E}$ :

$W_{E}=\frac{\Gamma}{\omega / c}, \quad W_{E} W_{H}=1$.

The field amplitude of the E-wave is determined by similar expressions [31].

Let us determine the ellipticity acquired by a linearly polarized test beam $(U=1)$ propagating in a waveguide in parallel to its axis. For the comparison to be convenient, let us take the test beam wavelength $\lambda=1064 \mathrm{~nm}$ and the path length $L=$ $=164 \mathrm{~cm}$, which corresponds to the conditions of 

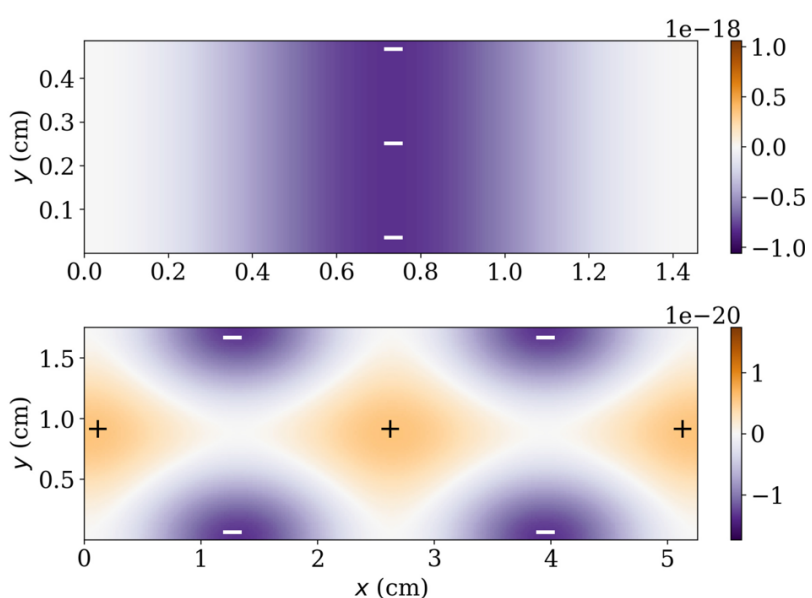

Fig. 7. Ellipticity of beams propagating along a waveguide at various points of its cross-section: $\mathrm{H}_{10}$ (upper panel) and $\mathrm{E}_{21}$ (bottom panel) waves

the PVLAS experiment. The parameters of a source producing microwaves that play the role of an external field are chosen proceeding from the characteristics of high-power klystrons developed at the SLAC center: a power of $75 \mathrm{MW}$ and the frequency $\nu=11.424 \mathrm{GHz}$. In order to determine field (19), the geometric dimensions of a waveguide are needed. Note that the parameters of the main wave $\mathrm{H}_{10}$ depend only on the side length $a$. As an illustration, let us put the side ratio $a: b=3: 1$. The actual size of side $a$ will be determined from the condition that the microwave operating frequency detuning from the critical waveguide frequency should be equal to $\left(\omega-\omega_{c}\right) / \omega=0.1$.

Figure 7 demonstrates the ellipticity of a test beam at various points of a waveguide cross-section. As one can see, the ellipticity magnitude is $10^{-18}$ by the order of magnitude, which is much worse than in the PVLAS experiment with a constant magnetic field. Such a discrepancy results from a low field induction in the waveguide, which amounts, in our case, to only about $0.2 \mathrm{~T}$. To achieve a value of $2.5 \mathrm{~T}$, the power $\bar{P}$ must reach a value of about $15 \mathrm{GW}$, which is now impossible from the practical viewpoint.

\section{Conclusions}

The effect of vacuum birefringence in an external field was theoretically predicted at the beginning of the twentieth century, but has not been observed experimentally till now. At present, the most exact exper- iment aimed at measuring the vacuum birefringence remains the PVLAS one $[14,15]$, in which a magnetic field of $2.5 \mathrm{~T}$ is used as an external one. High expectations are put on future laser installations, which should achieve the critical field strength value.

In this paper, we have considered a possibility to observe the effect in alternative configurations of an external field: in the field of a laser-driven coil generator and the field of a radio frequency waveguide. In the former case, the observed birefringence effect can be an order of magnitude larger than its value under the PVLAS experimental conditions. On the other hand, the destructive behavior of the generation can create practical difficulties and reduce the experimental accuracy. In the case where a microwave field and a waveguide are used, the effect is much lower for available microwave sources. The power that is required to enhance the effect to observable values is about $15 \mathrm{GW}$, which far exceeds current capabilities.

This work was sponsored by the grant of the Presidium of the National Academy of Sciences of Ukraine for Young Scientists (No. 0117U001760).

1. W. Heisenberg, H. Euler. Folgerungen aus der Diracschen Theorie des Positrons. Z. Phys. 98, 714 (1936).

2. A.E. Shabad. Photon dispersion in a strong magnetic field. Ann. Phys. 90, 166 (1975).

3. A.E. Shabad. Polarizaton of vacuum and quantum relativistic gas in an external field. Trudy Fiz. Inst. Akad. Nauk SSSR 192, 5 (1988) (in Russian).

4. A.E. Shabad. Photon propagation in a supercritical magnetic field. J. Exp. Theor. Phys. 98, 186 (2004).

5. A.E. Shabad, V.V. Usov. Real and virtual photons in an external constant electromagnetic field of most general form. Phys. Rev. D 81, 125008 (2010).

6. R.P. Mignani, V. Testa, D. González Caniulef, R. Taverna, R. Turolla, S. Zane, K. Wu. Evidence for vacuum birefringence from the first optical-polarimetry measurement of the isolated neutron star RX J1856.5-3754. Mon. Not. Roy. Astron. Soc. 465, 492 (2017).

7. J.S. Heyl, N.J. Shaviv. Polarization evolution in strong magnetic fields. Mon. Not. Roy. Astron. Soc. 311, 555 (2000).

8. J.S. Heyl, N.J. Shaviv. QED and the high polarization of the thermal radiation from neutron stars. Phys. Rev. D 66, 023002 (2002).

9. M. van Adelsberg, D. Lai. Atmosphere models of magnetized neutron stars: QED effects, radiation spectra and polarization signals. Mon. Not. Roy. Astron. Soc. 373, 1495 (2006).

ISSN 2071-0194. Ukr. J. Phys. 2018. Vol. 63, No. 11 
10. R. Fernández, S.W. Davis, The X-ray polarization signature of quiescent magnetars: effect of magnetospheric scattering and vacuum polarization. Astrophys. J. 730, 131 (2011).

11. R. Taverna, R. Turolla, D. González Caniulef, S. Zane, F. Muleri, P. Soffitta. Polarization of neutron star surface emission: a systematic analysis. Mon. Not. Roy. Astron. Soc. 454, 3254 (2015).

12. D. González Caniulef, S. Zane, R. Taverna, R. Turolla, $\mathrm{K}$. Wu. Polarized thermal emission from $\mathrm{X}$-ray dim isolated neutron stars: the case of RX J1856.5-3754. Mon. Not. Roy. Astron. Soc. 459, 3585 (2016).

13. E. Zavattini, G. Zavattini, G. Ruoso, G. Raiteri, E. Polacco, E. Milotti, V. Lozza, M. Karuza, U. Gastaldi, G. Di Domenico, F. Della Valle, R. Cimino, S. Carusotto, G. Cantatore, M. Bregant. New PVLAS results and limits on magnetically induced optical rotation and ellipticity in vacuum. Phys. Rev. D 77, 032006 (2008).

14. F. Della Valle, E. Milotti, A. Ejlli, G.M.L. Piemontese, G. Zavattini, U. Gastaldi, R. Pengo, G. Ruoso. First results from the new PVLAS apparatus: A new limit on vacuum magnetic birefringence. Phys. Rev. D 90, 092003 (2014).

15. F. Della Valle, A. Ejlli, U. Gastaldi, G. Messineo, E. Milotti, R. Pengo, G. Ruoso, G. Zavattini. The PVLAS experiment: Measuring vacuum magnetic birefringence and dichroism with a birefringent Fabry-Perot cavity. Eur. Phys. J. C 76, 24 (2016).

16. S. Shakeri, S.Z. Kalantari, S.-S. Xue. Polarization of a probe laser beam due to nonlinear QED effects. Phys. Rev. A 95, 012108 (2017).

17. A. Kosowsky. Cosmic microwave background polarization. Ann. Phys. 246, 49 (1996).

18. H. Kubo, R. Nagata. Stokes parameters representation of the light propagation equations in inhomogeneous anisotropic, optically active media. Opt. Commun. 34, 306 (1980).

19. H. Kubo, R. Nagata. Vector representation of behavior of polarized light in a weakly inhomogeneous medium with birefringence and dichroism. J. Opt. Soc. Am. 73, 1719 (1983).

20. H. Kubo, R. Nagata. Vector representation of behavior of polarized light in a weakly inhomogeneous medium with birefringence and dichroism. II. Evolution of polarization states. J. Opt. Soc. Am. A 2, 30 (1985).

21. H.J. Schneider-Muntau, J. Toth, H.W. Weijers. Generation of the highest continuous magnetic fields. IEEE Trans. Appl. Supercond. 14, 1245 (2004).

22. H. Daido, F. Miki, K. Mima, M. Fujita, K. Sawai, H. Fujita, Y. Kitagawa, S. Nakai, C. Yamanaka, Generation of a strong magnetic field by an intense $\mathrm{CO}_{2}$ laser pulse. Phys. Rev. Lett. 56, 846 (1986).

23. H. Daido, K. Mima, F. Miki, M. Fujita, Y. Kitagawa, S. Nakai, C. Yamanaka. Ultrahigh pulsed magnetic field produced by a $\mathrm{CO}_{2}$ laser. Jpn. J. Appl. Phys. 26, 1290 (1987).
24. E.S. Zhivopistsev, A.G. Kamushkin, V.V. Korobkin, A.V. Morozov, S.L. Motylev. Generation of a mega-gauss magnetic field in a coil oscillator with laser-driven emf. Kvant. Elektron. 18, 1142 (1991) (in Russian).

25. C. Courtois, A.D. Ash, D.M. Chambers, R.A.D. Grundy, N.C. Woolsey. Creation of a uniform high magneticfield strength environment for laser-driven experiments. J. Appl. Phys. 98, 054913 (2005).

26. G. Liao, Y. Li, B. Zhu, Y. Li, F. Li, M. Li, X. Wang, Z. Zhang, S. He, W. Wang, F. Lu, F. Zhang, L. Yang, K. Zhou, N. Xie et al. Proton radiography of magnetic fields generated with an open-ended coil driven by high power laser pulses. Matter Radiat. Extrem. 1, 187 (2016).

27. K.F.F. Law, M. Bailly-Grandvaux, A. Morace, S. Sakata, K. Matsuo, S. Kojima, S. Lee, X. Vaisseau, Y. Arikawa, A. Yogo, K. Kondo, Z. Zhang, C. Bellei, J.J. Santos, S. Fujioka, H. Azechi. Direct measurement of kilo-tesla level magnetic field generated with laser-driven capacitorcoil target by proton deflectometry. Appl. Phys. Lett. 108, 091104 (2016).

28. C. Rizzo, A. Rizzo, D.M. Bishop. The Cotton-Mouton effect in gases: experiment and theory. Int. Rev. Phys. Chem. 16, 81 (1997).

29. H.-H. Mei, W.-T. Ni, S.-J. Chen, S.-S. Pan. Axion search with Q \& A experiment. Mod. Phys. Lett. A 25, 983 (2010).

30. D. Sprehn, G. Caryotakis, A. Haase, E. Jongewaard, L. Laurent, C. Pearson, R. Phillips. Latest results in SLAC 75 MW PPM klystrons. In Proceedings of 7th Workshop on High Energy Density and High Power RF, Kalamata, Greece, 3-17 June, 2005, (American Institute of Physics, 2006), Vol. 807, p. 137.

31. V.V. Nikol'skii, T.I. Nikol'skaya, Electrodynamics and Propagation of Radio Waves (Nauka, 1989) (in Russian).

Received 30.10.18.

Translated from Ukrainian by O.I. Voitenko

\section{О. Новак, М. Дяченко, О. Падусенко, Р. Холодов}

ПОДВІЙНЕ ПРОМЕНЕЗАЛОМЛЕННЯ ВАКУУМУ В ПОЛІ КІЛЬЦЯ ЗІ СТРУМОМ ТА НАПРАВЛЕНОЇ ЕЛЕКТРОМАГНІТНОЇ ХВИЛІ

$\mathrm{P}$ е $з$ ю м е

Теоретично розглянуто ефект подвійного променезаломлення вакууму в зовнішньому полі, створеному кільцевим генератором магнітного поля з лазерною ЕРC, а також в полі направленої хвилі в прямокутному хвилеводі. Розраховано еліптичність, якої набуває лінійно поляризований лазерний промінь, що поширюється в полях вказаної конфігурації. Одержані результати порівнюються з характеристиками сучасного експерименту PVLAS, метою якого є спостереження ефекту подвійного променезаломлення вакууму в магнітному полі. 